\title{
Electroacupuncture promotes neural cell proliferation in vivo through activation of the ERK1/2 signaling pathway
}

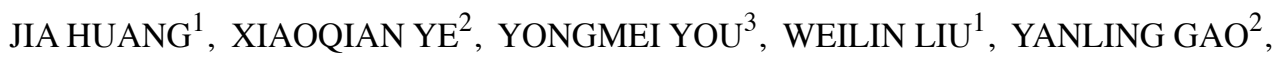 \\ SHANLI YANG ${ }^{3}, \mathrm{JUN} \mathrm{PENG}^{4}$, ZHENFENG HONG $^{4}, \mathrm{JING} \mathrm{TAO}^{1}$ and LIDIAN CHEN ${ }^{1}$ \\ ${ }^{1}$ College of Rehabilitation Medicine, ${ }^{2}$ MOE Key Laboratory of Traditional Chinese Medicine on Osteology and Traumatology \\ and Exercise Rehabilitation, ${ }^{3}$ Fujian Key Laboratory of Exercise Rehabilitation, ${ }^{4}$ Academy of Integrative Medicine, \\ Fujian University of Traditional Chinese Medicine, Fuzhou, Fujian 350122, P.R. China
}

Received January 23, 2014; Accepted March 10, 2014

DOI: $10.3892 /$ ijmm.2014.1702

\begin{abstract}
The aim of this study was to investigate the effect of electroacupuncture (EA) on cell proliferation and its molecular mechanisms. Sixty rats were randomly divided into 5 groups: sham operation control (SC), ischemia control (IC), EA, EA and DMSO injection (ED), EA and U0126 injection (EU). All the groups, with the exception of SC, underwent middle cerebral artery occlusion (MCAO), and DMSO or U0126 was injected into the rat in the ED or EU group 30 min prior to MCAO. Cell proliferation was evaluated by proliferating cell nuclear antigen (PCNA) immunostaining. The changes of cell cycle proteins (cyclin D1, CDK4, cyclin E, CDK2, p21 and p27) and the ERK1/2 pathway activation were examined by RT-PCR and western blot analysis. The results showed that the positive cell numbers of PCNA immunostaining in the EA and ED groups were more than those in the IC group $(\mathrm{P}<0.05)$. The mRNA and protein levels of p21 or p27 were obviously increased, however, the mRNA and protein levels of cyclin D1, CDK4, cyclin E and CDK2 were reduced in the IC and EU groups. The findings suggested that EA activates the ERK1/2 signaling pathway to protect brain injury during cerebral ischemia. However, this positive effect of EA can be blocked by U0126.
\end{abstract}

\section{Introduction}

Ischemic stroke is the most common type of stroke, accounting for $60-80 \%$ of all types of strokes (1). In an ischemic stroke, impairment of cerebral circulation causes hypoxia and ischemia, leading to localized ischemic necrosis or softening of

Correspondence to: Dr Lidian Chen or Dr Jing Tao, College of Rehabilitation Medicine, Fujian University of Traditional Chinese Medicine, 1 Huatuo Road, Minhou Shangjie, Fuzhou, Fujian 350122, P.R. China

E-mail: cld@fjtcm.edu.cn

E-mail: taojing01@163.com

Key words: electroacupuncture, ERK1/2 signaling pathway, ischemic stroke, cell proliferation brain tissue, as manifested by localized or diffuse loss of brain function. Restoring blood supply to ischemic brain tissue is the primary treatment for ischemic stroke and is performed via two main methods: non-drug therapy (e.g., extracranial - intracranial arterial anastomosis) and drug therapy (e.g., thrombolytic therapy). However, in recent years clinicians have found that in some patients with ischemic stroke, restoring blood supply to the brain tissue aggravates the symptoms instead of relieving them. Other studies have shown that the main pathological factors that contribute to such reperfusion injuries include an increased number of free radicals, calcium overload, excitatory amino acid toxicity and excessive apoptosis of neural cells $(2,3)$.

After the occurrence of acute brain ischemia, a penumbra zone develops around the lesion area. The metabolism and function of neurons in this zone are inhibited. If intervention can reverse such inhibition and activate these neurons, normal function can be restored. Animal studies have shown that $24 \mathrm{~h}$ after the occurrence of brain ischemia, neuronal axon initiation is triggered. The axons continue to grow and remodel over the next 14 days and reach maturity on day 28 . Intervention that is provided during neuronal axon initiation may be conducive to recovery of neurological functions $(4,5)$. In China, electroacupuncture is used to treat various diseases, including symptoms of stroke dysfunction. In western countries, the role of electroacupuncture has also been gradually recognized and promoted.

In the central nervous system of adult mammals, there are two types of special neuron clusters: the subependymal zone of the lateral ventricle and the subgranular zone of the hippocampal dentate gyrus. Under certain conditions, neurons in these zones are capable of proliferating (6). However, the amount of endogenous neural cell proliferation is limited and is not sufficient to counteract the brain damage induced by cerebral ischemia-reperfusion (I/R). Therefore, identifying drugs or measures that can stimulate endogenous neural stem cell proliferation and differentiation is of great importance.

The extracellular signal-regulated kinase 1/2 (ERK1/2) signaling pathway belongs to the family of mitogen-activated protein kinase signal transduction pathways. An abnormality in the ERK1/2 signaling pathway is closely associated with the occurrence and development of I/R injury. In neurons affected by ischemic stroke, various mechanisms lead to excessive 
activation of ERK, which then promotes cell proliferation, inhibits apoptosis, promotes cell invasion and affects cell differentiation.

In the present study, U0126, a highly selective ERK1/2 pathway inhibitor, was injected in an ischemic-reperfusion rat model and the mechanism underlying the neuroprotective function of electroacupuncture at acupoints of Zusanli (ST36) and Quchi (LI11), was explored. The results indicated that improvement in the neurological function of ischemic-reperfusion rats, as a result of electroacupuncture, is closely associated with the ERK1/2 pathway.

\section{Materials and methods}

Reagents. The reagents used in this study were: Cyclin D, cyclin-dependent kinase 4 (CDK4), cyclin E, CDK2, proliferating cell nuclear antigen (PCNA), p21, p27, $\beta$-actin primer (Shanghai Sangon Biological Engineering Technology and Services Co., Ltd., Shanghai, China), TRIzol (Life Technologies Co., Paisley, UK), TTC stain (Sigma, St. Louis, MO, USA), ERK1/2, p-ERK1/2, cyclin D, CDK4, CDK2, PCNA, p21, p27, anti- $\beta$-actin all rabbit antibody, rat anti-cyclin E antibody (Cell Signaling Technology, Inc., Danvers, MA, USA), dimethyl sulfoxide (DMSO) (Xiamen Lulong Biological Technology Development Co., Ltd., Beijing, China), and inhibitor U0126 (1,4-diamino-2,3-dicyano-1,4-bis[2-aminophenylthio]butadiene) (Promega Corp., Madison, WI, USA).

Instruments. Instruments used in this study included the Canon sx20 digital camera, gel preparation device, protein electrophoresis system, wet electroblotting system, gel imaging system, chemiluminescence imager, PCR electrophoresis system (Bio-Rad Corp., Hercules, CA, USA), automated microplate reader (BioTek, Hialeah, FL, USA), inverted microscope (Leica Corp., Germany), single-arm digital stereotaxic apparatus 68000 (RWD Life Technology Co., Ltd., China), ST-53311 syringe pump (Stoelting Corp., Wood Dale, IL, USA), and $50 \mu \mathrm{l}$ flat head micro-syringe (Hamilton Corp., Bonaduz, Switzerland).

Animal grouping. A total of 60 healthy, SPF grade, male, Sprague-Dawley (SD) rats, weighing $250 \pm 30 \mathrm{~g}$ and aged $2.21 \pm 0.15$ months, were purchased from the Shanghai SLAC Laboratory Animal Co., Ltd. [license number: SCXX (Shanghai) 2007-0005]. A random number table was used to divide the 60 rats into five groups of 12 rats each: the sham control group (SC), middle cerebral artery occlusion (MCAO) ischemic model control group (IC), MCAO + electroacupuncture group (EA), MCAO + electroacupuncture + DMSO group (ED) and MCAO + electroacupuncture + DMSO + U0126 group (EU). There was no significant difference in the weight or age of rats between the groups $(\mathrm{P}>0.05$, Table I).

Inhibitor and solvent administration. The U0126 inhibitor was diluted to $10 \mu \mathrm{M}$ and injected into the left ventricle of the rat stereotaxically (7). The dose used was based on relevant literature (8). Specifically, DMSO was used to dilute the U0126 inhibitor to $10 \mu \mathrm{M}$ and $5 \mu \mathrm{l}$ of the diluted $\mathrm{U} 0126$ inhibitor was loaded into the micro-syringe. Rats were anesthetized with $10 \%$ chloral hydrate (3 $\mathrm{ml} / \mathrm{kg}$ i.p.) after which, the parietal
Table I. Comparison in weight or age of rats between groups.

\begin{tabular}{lccc}
\hline Group & No. & Weight $(\mathrm{g})$ & Age (month) \\
\hline SC & 12 & $252.71 \pm 0.40$ & $2.11 \pm 0.41$ \\
IC & 12 & $258.67 \pm 0.39$ & $2.17 \pm 0.26$ \\
EA & 12 & $254.22 \pm 0.36$ & $2.15 \pm 0.30$ \\
ED & 12 & $255.19 \pm 0.36$ & $2.13 \pm 0.21$ \\
EU & 12 & $257.58 \pm 0.36$ & $2.14 \pm 0.33$ \\
\hline
\end{tabular}

skin was prepared and the surgical area disinfected via a strict protocol. Rats were then fixed in a stereotaxis and a skin incision was made along the parietal midline, exposing the anterior fontanelle, which was then marked. The lateral ventricle was precisely located (9) using the stereotaxis, at $2 \mathrm{~mm}$ lateral to and $1 \mathrm{~mm}$ below, the anterior fontanelle on the left side. Using the stereotaxis as a guide, a hole was drilled in the skull at this localized point and a needle attached to a micro-syringe was vertically inserted into the cerebral cortex to a depth of $\sim 3.5 \mathrm{~mm}$ beneath the surface. After confirmation that the needle tip was inside the ventricle, the syringe pump was turned on and the drug was slowly injected. Three min after completion of the injection, the micro-syringe was slowly retracted (the duration of needle retraction was $\geq 3 \mathrm{~min}$ ) and the skin was sutured. Drug administration was carried out $30 \mathrm{~min}$ prior to model establishment (7).

MCAO model. The animals were preoperatively fasted for $12 \mathrm{~h}$. Rats were weighed and anesthetized with an i.p. injection of $3 \mathrm{ml} / \mathrm{kg}$ chloral hydrate. Longa's suture-occluded method (10) was used to establish the MCAO and reperfusion model: in rats, the left middle cerebral artery area was selected as the infarction region and the detailed steps that were used are the same as those described in previous studies (11). Reperfusion was performed $2 \mathrm{~h}$ after occlusion and the suture was gently retracted to the carotid artery bifurcation. Intraoperative and postoperative room temperature was maintained at $\sim 25^{\circ} \mathrm{C}$. Incandescent light was used to keep the animal warm in order to maintain rectal temperature at $37 \pm 1^{\circ} \mathrm{C}$ until recovery as evidenced by resumption of normal activity. In the SC group, the artery was isolated, but not tied with suture. After surgery and recovery, the animals were placed in an environment at room temperature $\left(25^{\circ} \mathrm{C}\right)$ and resumed a normal diet.

Electroacupuncture intervention. Preparation for the electroacupuncture intervention involved the following: a) SC group: the animals were returned to the cage following the surgery and maintained, with no treatment being provided. b) IC group: the animals were returned to the cage after model establishment and maintained, with no treatment being provided. c) EA group: on the diseased side, Zusanli and Quchi acupoints were localized using the method described in 'experimental acupuncture' (12). A electroacupuncture instrument (G6805; Shanghai Huayi Co., Shanghai, China; peak voltage $=6 \mathrm{~V}$, dilatational wave, frequency $1-20 \mathrm{~Hz}$ ) was used and adjusted in order that the limbs were gently shaking. Each electroacupuncture session lasted $30 \mathrm{~min}$ and was carried out once a day, beginning the day after surgery and ending on the third day when the animals were euthanized. 
Animal sample collection. Animals in different groups were anesthetized, the abdominal aorta clamped and perfusion was performed through the left ventricle with $0.9 \%$ saline, followed by $4 \%$ paraformaldehyde in phosphate-buffered saline for fixation. The skull was opened and the brain was quickly removed and placed on ice. The whole brain tissue was placed in $4 \%$ paraformaldehyde in phosphate-buffered saline, frozen and stored at $4^{\circ} \mathrm{C}$. The tissues were embedded in paraffin for immunohistochemical testing. For TTC staining, animals in different groups were anesthetized, the abdominal aorta clamped and perfusion was performed through the left ventricle with $0.9 \%$ saline, followed by $4 \%$ paraformaldehyde in phosphate-buffered saline for fixation. The skull was opened and the brain was quickly removed and placed on ice. Whole brain tissue was placed in a $-80^{\circ} \mathrm{C}$ freezer. For western blotting and PCR, animals in different groups were anesthetized, the skull opened and the brain quickly removed and placed on ice. Cortical tissues from the left side were collected, using a scalpel, placed in Eppendorf PCR (EP) tubes and stored in liquid nitrogen for subsequent use.

Neurological severity score. The five-point scoring system developed by Longa et al (10) and Bederson et al (13) was used to assess the neurological severity of the model animals. The evaluation was performed $2 \mathrm{~h}$ after reperfusion and on days 1-3 immediately prior to electroacupuncture intervention. Neural damage in model rats that manifested $2 \mathrm{~h}$ after the establishment of infarction was scored on a scale of 0-4 points with a score of 1-3 points suggesting that the model was successful. Specifically the scores were: 0 points, no neurological deficit; 1 point, the tail was lifted and adduction (not able to fully extend) of the right forelimb was observed; 2 points, spontaneous circling to the right when walking; 3 points, the body was slanted to the right when walking; 4 points, not able to walk spontaneously along with possible loss of consciousness.

TTC staining. Fresh brain tissue was placed in $-80^{\circ} \mathrm{C}$ freezer for $20 \mathrm{~min}$, and sections were cut to a thickness of $\sim 2 \mathrm{~mm}$, along the coronal plane, starting $\sim 2 \mathrm{~mm}$ away from the end of the frontal lobe. Each brain sample was cut into 5-6 slices, which were placed in TTC, covered with aluminum foil and placed in an incubator at $37^{\circ} \mathrm{C}$ for $15-30 \mathrm{~min}$. The brain slices were turned frequently to achieve uniform staining. Following staining, the infarction region was stained white, whereas the other regions were stained red. The stained brain slices were placed on a scale, and images were captured. The Image-ProPlus image analysis and processing system were used to calculate the total volume of infarcted brain tissue in each sample. This volume was then divided by the total brain volume to obtain the percentage of brain infarction for subsequent statistical analysis.

Immunohistochemistry. The tissue was subjected to routine paraffin-embedded sectioning and antigen retrieval, then rinsed with buffer. Anti-PCNA primary antibody was then added in drops, as described in previous studies $(11,14)$. Under a x400 light microscope (Leica DM2500M; Leica Microsystems, Wetzlar, Germany) five non-overlapping fields were randomly selected and the number of positively stained cells was counted using automation.
Western blotting. A 200-mg sample of cortical tissue was collected from the left side of the brain. SDS-PAGE electrophoresis and membrane transfer were performed, the polyvinylidene fluoride membrane was removed, and then blocked with $5 \%$ skim milk for $2 \mathrm{~h}$. Anti-ERK1/2, p-ERK1/2, cyclin D, CDK4, cyclin E, CDK2, PCNA, p21 or p27 antibody was then added and the polyvinylidene fluoride was incubated at $4^{\circ} \mathrm{C}$ overnight. A corresponding secondary antibody $(1: 5,000)$ was then added and a computer scan was performed and the data stored for subsequent analysis.

Reverse transcription (RT)-PCR. RT-PCR was performed to determine mRNA expression levels of cyclin D, CDK4, cyclin E, CDK2, PCNA, p21 and p27 in the cortical tissues of left brain of rat. RNA extraction and PCR products were obtained as described in previous studies $(11,12)$. Then, $8 \mu 1$ of the PCR product was subjected to $1.5 \%$ agarose gel electrophoresis. Image analysis software was used to analyze the electrophoretic bands, and the integrated density value (IDV) of each band was obtained.

Statistical analysis. SPSS 16.0 was used for statistical analysis. Data are expressed as mean \pm standard deviation. For multiple comparisons of quantitative data, one-way analysis of variance (ANOVA) was used. If the data satisfied homogeneity of variance, the Fisher's least significant difference (LSD) method was applied, otherwise Tamhane's method was applied. $\mathrm{P}<0.05$ was considered statistically significant.

\section{Results}

Neurologicalassessmentand brain tissue morphologyof MCAO rats following electroacupuncture treatment. Assessment of the neurological behavior of rats showed no significant difference between the IC and EU groups at $2 \mathrm{~h}, 1-3$ days after the reperfusion ( $\mathrm{P}>0.05)$. In the EA and ED groups, as disease progressed and the electroacupuncture intervention proceeded, the neurological scores gradually decreased. The score 2 days after reperfusion was lower compared with $2 \mathrm{~h}(\mathrm{P}>0.05)$ and 1 day $(\mathrm{P}<0.05)$. The score was lowest 3 days following reperfusion $(\mathrm{P}<0.05)$. No significant differences were observed between any of the groups at $2 \mathrm{~h}$ and 1 day after reperfusion $(\mathrm{P}>0.05)$. No significant differences were identified between the IC and EU groups or between the EA and ED groups $(\mathrm{P}>0.05)$ at $2 \mathrm{~h}$ and 2 days after reperfusion. The neurological scores of the EA and ED groups were significantly lower compared to the IC group $(\mathrm{P}<0.05)$. However, the neurological score of the EU group was higher compared to the EA and ED groups ( $\mathrm{P}>0.05)$ likely due to the effect of U0126 (Table II).

For the morphological and histological analyses, brain slices of MCAO model rats were subjected to TTC staining and examined. The IC group exhibited the largest volume of cerebral infarction $(28.67 \pm 5.79, n=3)$. The volume of cerebral infarction was significantly smaller in the EA $(13.60 \pm 1.71, n=3)$ and ED $(14.74 \pm 1.04, n=3)$ groups compared with the IC group $(\mathrm{P}=0.001)$. The cerebral infarction volume was also smaller in the EU $(23.69 \pm 3.04, n=3)$ compared with the IC group, however the difference was not significant $(\mathrm{P}=0.113)$. Similarly, there was no significant difference in cerebral infarction volume when comparing the EA and ED groups $(\mathrm{P}=0.695)$. 
A

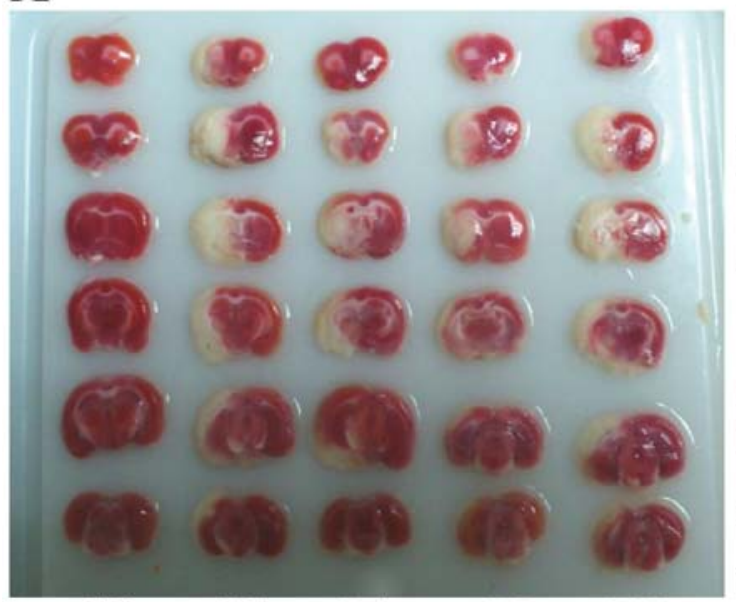

SC

IC

EA

ED

EU
B

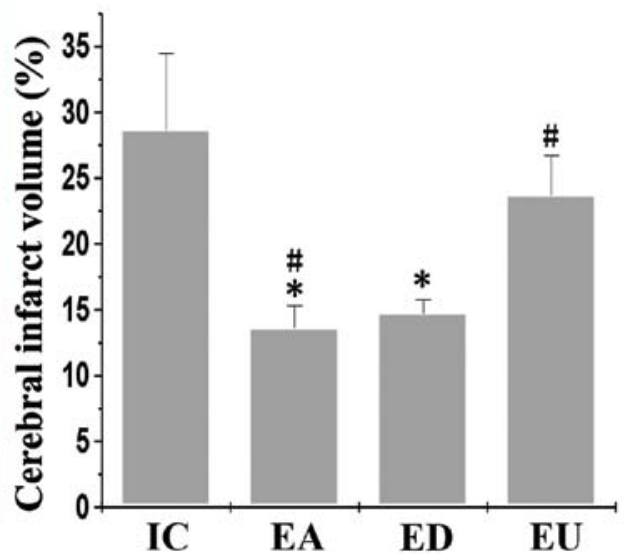

Figure 1. The neurological protective effect of EA on brain damage in rats. (A) TTC staining of brain tissue. After TTC (3,5-triphenyl tetrazolium chloride) staining, normal brain is red while the cerebral infarction region is white. (B) Analysis of TTC staining. The value is the ratio of cerebral infarct volume (CIV) to total brain volume ( $\mathrm{n}=3)$. ${ }^{*} \mathrm{P}<0.05$ vs. IC and EU; ${ }^{*} \mathrm{P}>0.05$ IC vs. EU, EA vs. ED.
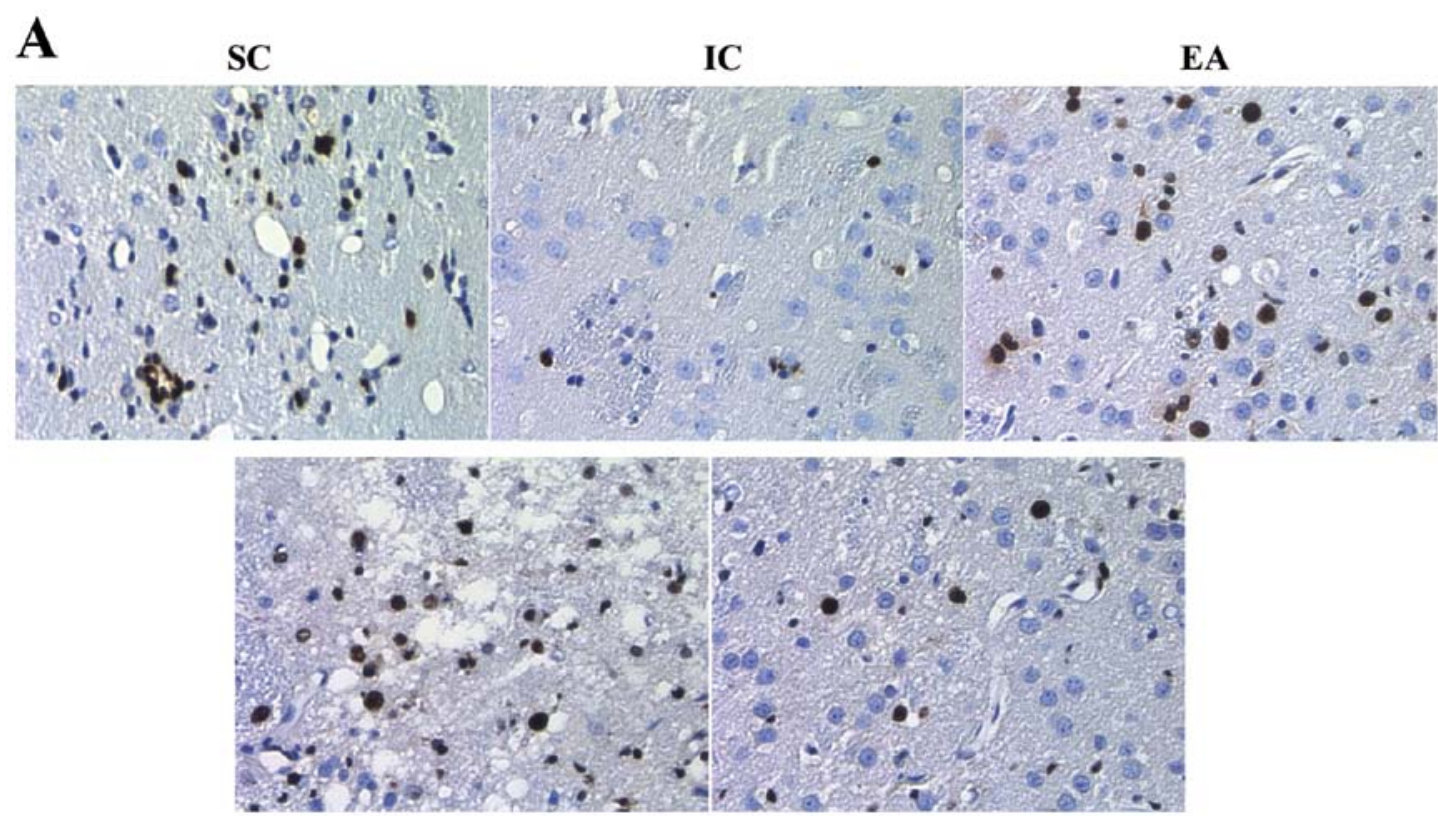

ED

EU

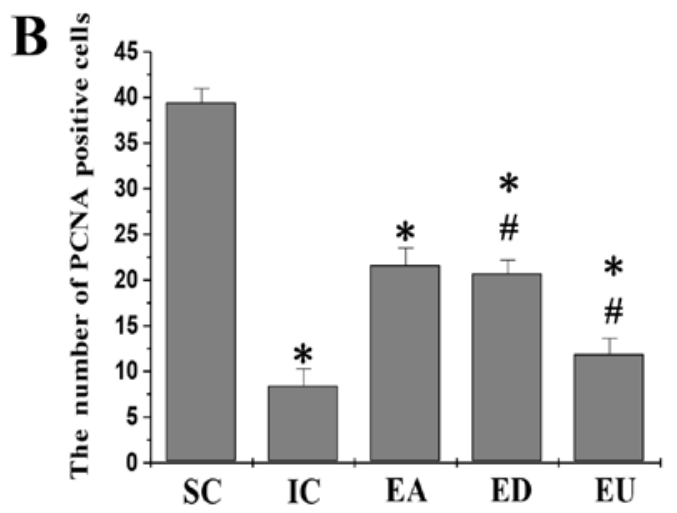

C

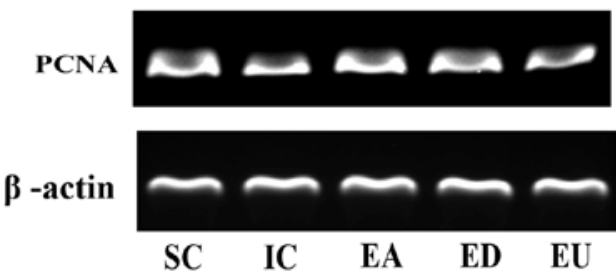

Figure 2. EA treatment upregulates PCNA expression 3 days after I/R in rats, which effect is blocked by ERK1/2 inhibitor, U0126. (A) Immunostaining PCNA in brain tissues from different groups. Scale bar, $400 \mu \mathrm{m}$. (B) Treatment with EA significantly increased the number of PCNA ( $=3$ ). ${ }^{*} \mathrm{P}<0.05 \mathrm{vs}$. SC; ${ }^{*} \mathrm{P}<0.05$ vs. IC and EU; ${ }^{\prime P}>0.05$ IC vs. EU, EA vs. ED. (C) The mRNA expression of PCNA was determined by RT-PCR. $\beta$-actin was used as internal control. 
Table II. Neurological deficit scores.

\begin{tabular}{lcccc}
\hline $\begin{array}{l}\text { Groups } \\
(\mathrm{n}=12)\end{array}$ & $\begin{array}{c}2 \mathrm{~h} \\
\text { after I/R }\end{array}$ & $\begin{array}{c}1 \text { day } \\
\text { after I/R }\end{array}$ & $\begin{array}{c}2 \text { days } \\
\text { after I/R }\end{array}$ & $\begin{array}{c}3 \text { days } \\
\text { after I/R }\end{array}$ \\
\hline SC & 0 & 0 & 0 & 0 \\
IC & $2.67 \pm 0.39$ & $2.71 \pm 0.40$ & $2.58 \pm 0.42$ & $2.33 \pm 0.49$ \\
EA & $2.58 \pm 0.36^{\mathrm{a}}$ & $2.50 \pm 0.30$ & $1.92 \pm 0.60$ & $1.42 \pm 0.29^{\mathrm{b}, \mathrm{c}}$ \\
ED & $2.58 \pm 0.47^{\mathrm{a}}$ & $2.54 \pm 0.33$ & $2.04 \pm 0.36$ & $1.38 \pm 0.31^{\mathrm{b}}$ \\
EU & $2.63 \pm 0.38^{\mathrm{a}}$ & $2.67 \pm 0.44$ & $2.42 \pm 0.36$ & $2.29 \pm 0.40$ \\
\hline
\end{tabular}

Ischemia-reperfusion (I/R): ${ }^{\mathrm{a}} \mathrm{P}>0.05$ vs. IC and $\mathrm{EU} ;{ }^{\mathrm{b}} \mathrm{P}<0.05$ vs. IC and $\mathrm{EU}$; ${ }^{\mathrm{C}}>0.05$ vs. ED.

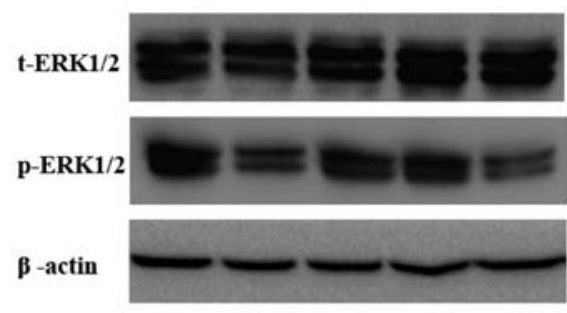

SC IC EA ED EU

Figure 3. Effect of EA treatment and U0126 on the activation of ERK1/2 signaling pathway. EA treatment increased the phosphorylation levels of ERK1/2 (p-ERK1/2), blocked by U0126. Total protein levels of ERK1/2 (t-ERK1/2) were not affected by I/R or EA treatment. $\beta$-actin was used as the internal control.

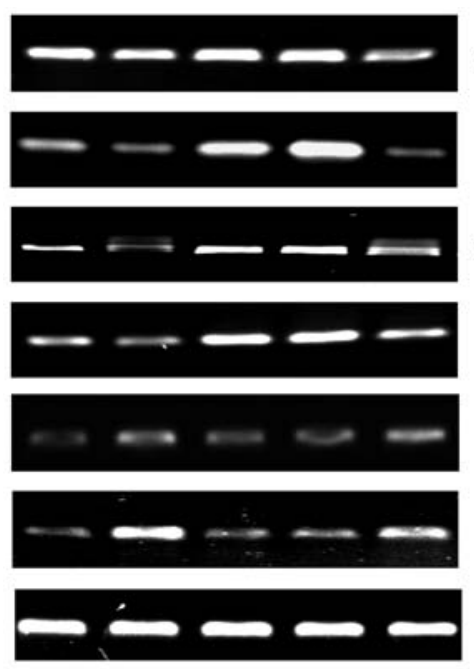

SC IC EA ED EU

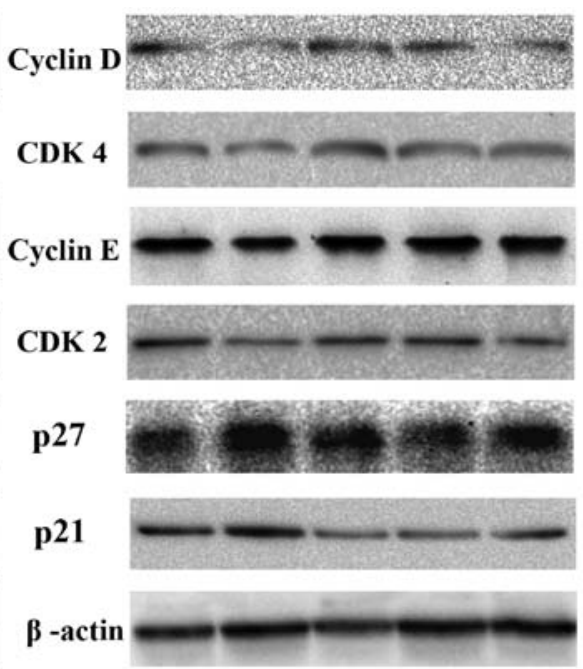

SC IC EA ED EU

Figure 4. The mRNA (left) and protein (right) expression of cyclin D1, CDK4, cyclin E, CDK2, p21Cip1 and p27Kip1 in the left brain cortex 3 days after I/R in different groups. EA promoted the expression of cell cycle-positive regulation factors, but restrained the cell cycle inhibitors.

Neurological score and the TTC staining results suggest that electroacupuncture intervention treatment is effective and that adding inhibitors can reduce treatment efficacy (Fig. 1).

PCNA expression in cerebral ischemia and reperfusion model rats. In immunohistochemical testing, PCNA-positive substances that are located in the nuclei are brown in color and serve as a good indicator of the state of cell proliferation. The number of PCNA-positive cells was reduced in the IC, EA, ED and EU groups (Fig. 2A). However, the number of PCNA positive cells was significantly higher in both the EA and ED groups compared with the IC group. No significant difference was observed in the number of PCNA-positive cells in the IC vs. EU group (Fig. 2B). To validate these results, RT-PCR was performed to evaluate PCNA expression. Results obtained by measuring PCNA mRNA expression showed the same trend as that determined using immunohistochemistry (Fig. 2C). This observation suggests that cell proliferation occurs after electroacupuncture in the infarction region of the rat cortex.

Electroacupuncture and p-ERK1/2 expression in cerebral ischemia and reperfusion model rats. As shown in Fig. 3,
t-ERK1/2 was not notably different among different groups. By contrast, p-ERK1/2 expression in the EA and ED groups was notably increased compared to the SC group, whereas p-ERK1/2 expression in the IC group was decreased compared to the SC group. p-ERK1/2 protein expression in the EU group was lower compared to the SC group due to the effect of U0126. These results suggested that in cerebral ischemia and reperfusion model rats, U0126 is able to block activation of the ERK1/2 signaling pathway induced by electroacupuncture, whereas DMSO does not have any notable effects on brain damage.

Analyses on growth factors promoting cell proliferation. As shown in Fig. 4, the mRNA, or transcription levels of cyclin D, CDK4, cyclin E, and CDK2 were notably higher in the EA and ED groups, and lower in the EU group, compared to the IC group. Protein expression levels of cyclin D, CDK4, cyclin E and CDK2 were consistent with the mRNA results. The mRNA and protein expression of the cyclin kinase inhibitors p21 and p27 was notably inhibited in the EA and ED groups, and notably increased in the EU group, compared to the IC group. This result suggested that, after electroacupuncture intervention, p21 and 
p27 expression is downregulated, although this effect can be reversed by U0126.

\section{Discussion}

Ischemic stroke belongs to the 'stroke' category in traditional Chinese medicine. According to the theory of traditional Chinese medicine, the basic mechanism of ischemic stroke is loss of ying and yang balance, as well as disruption of Qi and blood flow. Ancient Chinese physicians believed that paralysis should be treated 'through Yangming meridians'. Zusanli and Quchi are both in the Yangming meridians. Most Yangming meridians are full of Qi and blood flow; if Qi and blood flow are smooth in Yangming meridians, the whole meridian system is clear and this allows the body to recover. The primary lesion area in a stroke is the head. Quchi belongs to the hand Yangming meridian, and Zusanli belongs to the foot Yangming meridian. The hand and foot Yangming meridians pass through the head and the Qi in these two meridians can act together to treat stroke. Previous studies $(11,14)$ on a large amount of ancient Chinese medical literature conducted by our group have shown that Zusanli and Quchi are the two acupoints that have been used relatively frequently for stroke treatment. In rats with cerebral ischemia and reperfusion, electroacupuncture at Zusanli and Quchi was found to significantly increase $\beta$-EP and Glu expression levels in the hypothalamus, reduce the total calcium level and free radicals in the brain tissue and inhibit excitatory amino acid toxicity (15). Functional magnetic resonance imaging has also confirmed that acupuncture at Zusanli activates most brain regions, mainly the frontal lobe, temporal lobe, island lobe, parietal lobe, cingulate gyrus and cerebellum, and that temporal lobe activation was found to be the most substantial $(16,17)$. The results of the present study have demonstrated that the volume of the infarction region in the EA group was significantly smaller than that in the IC group $(\mathrm{P}<0.05)$. This finding suggests that electroacupuncture can reduce the infarct volume in cerebral $\mathrm{I} / \mathrm{R}$ rats, which is consistent with results of a previous study conducted by our group (11).

Neurological severity scores may be used to predict the histological effects in cerebral ischemia and reperfusion model rats (18). The results of the present study show that on the third day after reperfusion the neurological severity score of the EA group was significantly lower than that of the IC group $(\mathrm{P}<0.05)$. This suggests that 3 days of daily treatment with electroacupuncture effectively improves neurological function in rats that have had $2 \mathrm{~h}$ of ischemia followed by reperfusion. A previous study (19) demonstrated that in male, bilateral artery ischemia gerbils, p-ERK1/2 expression reached a peak value $5 \mathrm{~min}$ after reperfusion, following $3.5 \mathrm{~min}$ of ischemia and that on day 3 after reperfusion the p-ERK1/2 expression was no longer significantly different compared with the sham group $(\mathrm{P}>0.05)$. This finding suggests that reperfusion activates the ERK1/2 pathway (19). Since the ERK1/2 pathway appears to be deactivated by day 3 post-ischemia and reperfusion, we hypothesized that the neurological improvement observed on day 3 in the present study was due to activation of the ERK1/2 pathway by electroacupuncture in the EA group.

The ERK1/2 pathway is closely associated with ischemic stroke. However, whether it plays a protective or damaging role after ischemic stroke is still under debate (20). A large amount of evidence has shown that activation of the ERK1/2 pathway protects brain tissue after ischemic stroke. For instance after cerebral ischemia reperfusion injury, ERK1/2 can inhibit inflammatory reactions in the brain tissue (21), promote osteopontin (OPN) secretion, thereby reducing the release of excitatory amino acids (22), and enhance IL-20 expression, promoting cell proliferation, thus protecting ischemic brain tissue (23). PD98059 and U0126 are both effective ERK1/2 pathway inhibitors. PD98059 suppresses the activation of mitogen-activated protein kinase kinase 1 (MAP2K1, also known as MEK1) induced by upstream kinases, but is ineffective on MEK1 which has already been activated and activated MEK1 can still phosphorylate ERK1/2. In comparison, U0126 inhibits MEK1 and MEK2 highly selectively, whether activated or not, thus effectively suppressing ERK1/2 phosphorylation. Thus, the inhibitory effect on the ERK1/2 pathway of U0126 is more stable than that of PD98059 (24). Therefore, in the present study, U0126 was used as the ERK1/2 pathway inhibitor. The results of the present study have demonstrated that $\mathrm{p}$-ERK $1 / 2$ protein expression in the EA group was significantly higher than that in the SC and IC groups, suggesting that 3 days of electroacupuncture intervention following reperfusion after $2 \mathrm{~h}$ of ischemia activates the ERK1/2 pathway. Compared with the EA and ED groups, p-ERK protein expression in the EU group was significantly decreased, whereas p-ERK protein expression in the EA and ED groups did not differ significantly. This finding suggests that U0126 effectively inhibits ERK1/2 pathway activation and blocks the pathway activation effect of electroacupuncture.

The present study has demonstrated that electroacupuncture improved neurological function in I/R rats. In addition, the ERK1/2 signaling pathway is one of the key pathways that regulate cell proliferation. We therefore hypothesized that the effect of electroacupuncture may be associated with the regulation of cell proliferation. PCNA is a good indicator of cell proliferation (25). It can reflect the activity of neural cell proliferation, thus helping to promote nerve repair after brain injury (26). In the present study, immunohistochemistry and RT-PCR were performed to examine PCNA levels and the results showed that the number of PCNA-positive cells in the EA group was significantly higher than that in the IC and EU groups $(\mathrm{P}<0.05)$. This finding suggests that electroacupuncture increases PCNA expression and promotes cell proliferation, consistent with a previous finding (11).

Cell proliferation is mainly achieved through progression of cell cycles and is precisely regulated by three types of factors: CDKs, cyclins and CKIs. In cell cycles specific CDKs and specific cyclins bind, form complexes, and connect the signaling pathway with the cell cycle at the G1/S-phase transition. Studies have shown that when cells enter the S-phase from the G1-phase, the phosphorylation and activation of ERK1/2 pathway is crucial to inducing the binding of cyclin D1 and CDK4 and the binding of cyclin E and CDK2. U0126 inhibits ERK1/2 activation, thus effectively inhibiting the proliferative effect (27-29). When PD98059 inhibits ERK1/2 signaling pathway activation, p27Kip1 expression is upregulated, cyclin D1 and E expression is downregulated and the cells are arrested in the G1-phase (30). Enhancement of p21Cip1 and p27Kip1 expression suppresses the binding of cyclin $\mathrm{E}$ and $\mathrm{CDK} 2$ and hampers the progression of the cell cycle from the G1/G0 to the S-phase (28). The results of the present study show that electroacupuncture substantially 
upregulated the protein and gene expression of cyclin D1, $\mathrm{CDK} 4$, cyclin $\mathrm{E}$ and $\mathrm{CDK} 2$. Overexpression of these positive regulatory factors can shorten the G1-phase, cause the G0/S and/or G1/S transition point to be missed and lead to continued proliferation. Consistent with this, electroacupuncture intervention significantly reduces the gene and protein expression of negative regulators $\mathrm{p} 21 \mathrm{Cip} 1$ and $\mathrm{p} 27 \mathrm{Kip} 1$, thus effectively preventing their inhibitory effect on positive regulatory factors, helping to promote cell proliferation. The present study also shows that DMSO did not have the above effect. However, despite electroacupuncture intervention, cell proliferation in the EU group was not found to increase. Instead, a high expression of p21Cip1 and p27Kip1 inhibited the expression of cyclin D1, CDK4, cyclin E and CDK2 expression, the cell cycle was arrested in the G1-phase and the cells stopped growing, leading to inhibition of cell proliferation.

In conclusion, the present study shows that electroacupuncture at Zusanli and Quchi in cerebral I/R rats activates the ERK1/2 pathway, upregulates positive regulatory factors PCNA, cyclin D1, CDK4, cyclin E and CDK2 and downregulates negative regulatory factors $\mathrm{p} 21 \mathrm{Cip} 1$ and $\mathrm{p} 27 \mathrm{Kip} 1$. This allows rat cortical cells on the ischemic side to pass the G1-phase and enter the S-phase 3 days after $2 \mathrm{~h}$ of ischemia followed by reperfusion. This promotes neural cell proliferation and plays a protective role in the brain, thereby improving neurological function in the rats. Taken together, these data demonstrate that electroacupuncture treatment is an effective measure for treating ischemic stroke. However, the mechanism for long-term neuroprotective effects of electroacupuncture at Zusanli and Quchi should be further investigated.

\section{Acknowledgements}

We would like to thank Yijing Jiang, Yulong Zou, Zhicheng Lin and Jiumao Lin for assistance. This study was sponsored by the Natural Science Foundation of China (No. 81373778) and Medical Innovation Program of the Fujian Ministry of Health of China (No. 2012-CX-28).

\section{References}

1. The Chinese medical association of neurology, cerebrovascular epidemiology group of acute ischemic stroke treatment guidelines writing group: China's acute ischaemic stroke treatment guidelines. Chinese General Practice pp4013-4017, 2010.

2. Diener HC, Foerch C, Riess H, et al: Treatment of acute ischaemic stroke with thrombolysis or thrombectomy in patients receiving anti-thrombotic treatment. Lancet Neurol 12: 677-688, 2013.

3. Flynn RW, MacWalter RS and Doney AS: The cost of cerebral ischaemia. Neuropharmacology 55: 250-256, 2008.

4. Hong J, Wu G, Zou Y, et al: Electroacupuncture promotes neurological functional recovery via the retinoic acid signaling pathway in rats following cerebral ischemia-reperfusion injury. Int $\mathrm{J}$ Mol Med 31: 225-231, 2013.

5. Le W, Liu Y, Wang Q, et al: Effect of scalp-acupuncture on cerebral ischemia/reperfusion rats of proliferation and differentiation intervention neural stem cell. J Hubei Univ Trad Chin Med 2: 12-15, 2013.

6. Tao J, Xue XH, Chen LD, et al: Electroacupuncture improves neurological deficits and enhances proliferation and differentiation of endogenous nerve stem cells in rats with focal cerebral ischemia. Neurol Res 32: 198-204, 2010.
7. Kawano T, Fukunaga K, Takeuchi Y, et al: Neuroprotective effect of sodium orthovanadate on delayed neuronal death after transient forebrain ischemia in gerbil hippocampus. J Cereb Blood Flow Metab 21: 1268-1280, 2001

8. Pignataro G, Meller R, Inoue K, et al: In vivo and in vitro characterization of a novel neuroprotective strategy for stroke: ischemic postconditioning. J Cereb Blood Flow Metab 28: 232-241, 2008.

9. Bao XM and Shu SY: The stereotaxic atlas of the rat brain. People's Medical Publishing House pp7-35, 1991.

10. Longa EZ, Weinstein PR, Carlson S, et al: Reversible middle cerebral artery occlusion craniectomy in rats. Stroke 20: 84-91, 1989.

11. Xie G, Yang S, Chen A, et al: Electroacupuncture at Quchi and Zusanli treats cerebral ischemia-reperfusion injury through activation of ERK signaling. Exp Ther Med 5: 1593-1597, 2013.

12. Li ZR: The subject of experimental acupuncture and moxibustion. Chinese Press of Traditional Chinese Medicine, Beijing, 2003.

13. Bederson JB, Pitts LH, Tsuji M, et al: Rat middle cerebral artery occlusion: evaluation of the model and development of a neurologic examination. Stroke 17: 472-476, 1986.

14. Xue X, You Y, Tao J, Ye X, et al: Electro-acupuncture at points of Zusanli and Quchi exerts anti-apoptotic effect through the modulation of PI3K/Akt signaling pathway. Neurosci Lett 558: 14-19, 2014.

15. Cai Y-Y, Liu Z-S, Wang S, et al: The influence of electroacupuncture acupoints on the protein expression of $\beta$-EP and glu hypathalamus in rats with cerebral ischemia reperfusion injury. Chin J Bas Med Trad Chin Med 16: 1030-1033, 2010.

16. Xiao YY, Du L, Hong BK, et al: Study the acupuncture at acupoint of Zusanli effects in the brain magnetic resonance imaging. Sichuan Zhongyi 25: 98-101, 2007.

17. Wang W, Qi JP, Xia YL, et al: The response of human motor cortex to acupuncture of S36 and G34 as revealed by functional MRI. Chin J Phys Med Rehabil 26: 472-475, 2004.

18. Garcia JH, Wagner S, Liu KF and Hu XJ: Neurological deficit and extent of neuronal necrosis attributable to middle cerebral artery occlusion in rats. Statistical validation. Stroke 26: 627-634, 1995.

19. Namura S, Iihara K, Takami S, et al: Intravenous administration of MEK inhibitor U0126 affords brain protection against forebrain ischemia and focal cerebral ischemia. Proc Natl Acad Sci USA 98: 11569-11574, 2001.

20. Sawe N, Steinberg G and Zhao H: Dual roles of the MAPK/ERK1/2 cell signaling pathway after stroke. J Neurosci Res 86: 1659-1669, 2008.

21. Sironi L, Banfi C, Brioschi M, et al: Activation of NF- $\mathrm{KB}$ and ERK1/2 after permanent focal ischemia is abolished by simvastatin treatment. Neurobiol Dis 22: 445-451, 2006.

22. Meller R, Stevens SL, Minami M, et al: Neuroprotection by osteopontin in stroke. J Cereb Blood Flow Metab 25: 217-225, 2005.

23. Chen WY and Chang MS: IL-20 is regulated by hypoxia-inducible factor and up-regulated after experimental ischemic stroke. J Immunol 182: 5003-5012, 2009.

24. Dang ZC and Lowik CW: Differential effects of PD98059 and U0126 on osteogenesis and adipogenesis. J Cell Biochem 92: 525-533, 2004.

25. Kim TJ and Yun YP: Potent inhibition of serum-stimulated responses in vascular smooth muscle cell proliferation by 2-chloro3-(4-hexylphenyl)-amino-1,4-naphthoquinone, a newly synthesized 1,4-naphthoquinone derivative. Biol Pharm Bull 30: 121-127, 2007.

26. Wharton SB, Williams GH, Stoeber K, et al: Expression of Ki67, PCNA and the chromosome replication licensing protein Mcm2 in glial cells of the aeing human hippocampus increases with the burden of Alzheimer-type pathology. Neurosci Lett 383: 33-38, 2005.

27. Wang B, Gao Y, Xiao Z, et al: ERK1/2 prootes proliferation and inhibits neuronal differentiation of neural stem cells. Neurosci Lett 461: 252-257, 2009.

28. Feng Q, Huang S, Zhang A, et al: Y-box protein 1 stimulates mesangial cell proliferation via activation of ERK1/2. Nephron Exp Nephrol 113: e16-e25, 2009.

29. Osaki LH, Figueiredo PM, Alvares EP and Gama P: EGFR is involved in control of gastric cell proliferation through activation of MAPK and Src signaling pathways inearly-weaned rats. Cell Prolif 44: 174-182, 2011.

30. Sah JF, Balasubramanian S, Eckert RL and Rorke EA: Epigallocatechin-3-gallate inhibits epidermal growth factor receptor signaling pathway. Evidence for direct inhibition of ERK1/2 and AKT kinases. J Biol Chem 279: 12755-12762, 2004. 